\title{
LA VISIÓN SCHUMPETERIANA DE LA INNOVACIÓN. APORTACIONES Y CONTRADICCIONES
}

\author{
ANTONIO MARTÍNEZ GONZÁLEZ*
}

Fecha de recepción: 19 de abril de 2015.

Fecha de aceptación: 15 de diciembre de 2015.

I

\section{LA ECONOMÍA BASADA EN EL CONOCIMIENTO. DESDE LA COMPETENCIA ESTÁTICA A LA COMPETENCIA DINÁMICA}

La economía neoclásica desarrolla de manera formal la metáfora de la «mano invisible» de Adam Smith, según la cual los intereses de cada individuo convergen hacia el interés colectivo - es decir, el máximo bienestar - a través del mecanismo intangible de la competencia.

La economía neoclásica se basa en el concepto de equilibrio: el mercado en competencia perfecta garantiza la asignación óptima de los recursos y la coordinación de las decisiones de cada agente atomizado se alcanza por medio de los precios. En el equilibrio, todas las empresas son iguales y los beneficios extraordinarios son nulos, es decir, el rendimiento del capital, o beneficio normal, es igual a la tasa de interés. Al tratar este tipo de beneficio como un coste, se suele hablar de beneficios nulos, pero en realidad son los beneficios por encima de la tasa de interés - es decir, los beneficios extraordinarios- los que son nulos en el equilibrio.

Así pues, la teoría neoclásica tradicional se ocupa casi exclusivamente de la situación de equilibrio en competencia perfecta, en la

\footnotetext{
* Profesor Titular de Economía Aplicada y del Máster de Economía de la Escuela Austriaca, Universidad Rey Juan Carlos, Madrid. antonio.martinez@urjc.es 
cual se supone que todos los datos de los distintos agentes económicos se han coordinado y ajustado entre ellos, mientras que en la realidad, como indica Hayek (1945), el problema económico de la sociedad es esencialmente el de adaptarse rápidamente a los cambios que se producen en determinadas circunstancias de tiempo y espacio. Consecuentemente, la competencia es un proceso dinámico, cuyas características esenciales son ignoradas por las rígidas hipótesis que se derivan del análisis estático de origen neoclásico. El concepto de eficiencia dinámica hace referencia también a la evolución de los mercados en el tiempo, el contexto de una visión cíclica del desarrollo económico. Las variables del cambio tecnológico y de la innovación se vuelven endógenas, siendo directamente influenciadas por la actividad del empresario, cuyo papel es muy distinto del que éste desarrolla —anónimamente- en la teoría neoclásica.

El modelo neoclásico ignora por completo el problema del conocimiento y del aprendizaje. De hecho, todas las empresas son iguales porque todos los agentes tienen una información perfecta de todos los aspectos del mercado: precios vigentes de bienes y factores, funciones de costes y tecnología utilizada en la producción. Sin embargo, el hecho de compartir el conocimiento tecnológico es una hipótesis infundada en la práctica. Además, en este planteamiento, el conocimiento organizacional no tiene lugar, y simplemente se le ignora, en cuanto las empresas en competencia perfecta son simples funciones de producción, sin ninguna estructura organizativa. De esta forma, nadie se preocupa por lo que hacen sus competidores y cada empresa se limita a aplicar el algoritmo - conocido- que permite la maximización de los beneficios. Es evidente que si una empresa de un sector determinado dispone de las mismas informaciones, de los mismos factores de producción y de las mismas tecnologías de producción que las demás empresas y si aplica el mismo algoritmo $-P=C M_{g^{\prime}}$ precio igual a coste marginal- tendrá necesariamente que ser igual a las demás.

Los economistas de Escuela Austriaca —en particular gracias a Friedrich von Hayek (1945) — han conseguido superar las contradicciones del modelo neoclásico, al indicar que el sistema de precios es el mecanismo de transmisión de la información y el medio de interacción entre los agentes económicos, del cual surgen las mejores tecnologías, prácticas comerciales y modelos organizacionales, que 
no se conocen a priori, pero que los agentes tienen que descubrir y aprender. Los individuos y las empresas presentan limitaciones cognitivas y desarrollan competencias, habilidades y experiencias que son específicas y personales, en otras palabras, que son idiosincrásicas. Basándonos de este supuesto, el mercado proporciona señales por medio de los precios, que evidencian la existencia de diferencias en la forma de producir los bienes, sin por ello excluir el hecho de que los bienes son también señales de las necesidades de los consumidores y que por lo tanto regulan la coordinación espontánea y no planificada de la economía. Los agentes económicos obtienen así informaciones sobre los conocimientos relevantes, y el papel del mercado es, por ende, el de hacer posible la interacción entre las diferentes competencias y conocimientos.

Supongamos que el sistema económico se encuentre en una situación de equilibrio, con precios, tecnologías y preferencias constantes. Si de repente en el mercado de un determinado bien aparecen unos vendedores que ofrecen dicho bien a un precio más bajo, puesto que son capaces de producirlo de forma más eficiente, los compradores tenderán a recurrir a ellos. Los productores que no dispongan de la nueva técnica de producción verán cómo sus beneficios se reducen, e incluso podrían llegar a tener pérdidas. Para los empresarios, ésta es una señal muy precisa y ejerce una fuerte presión para adaptarse rápidamente a las nuevas formas productivas.

Así pues, el núcleo del problema es que, fuera del equilibrio, en el mercado no existe un único precio que funciona como señal de la relación entre oferta y demanda - escasez o excedente de un producto- sino precios diferentes determinados por los distintos productores, que serán por tanto fijadores de precios. En otras palabras, se hace hincapié en el proceso de ajuste que conlleva la introducción de nuevos conocimientos más que en el equilibrio Pareto-óptimo.

II

\section{LA REVOLUCIÓN SCHUMPETERIANA. LA INNOVACIÓN Y LA DINÁMICA DE LA DESTRUCCIÓN CREATIVA}

Schumpeter, conocedor del planteamiento austriaco de competencia dinámica, desarrolla su propia visión, según la cual la virtud 
del sistema de mercado no reside en la eficiencia estática - asignativa y productiva - sino en la innovación, es decir, la eficiencia dinámica. Schumpeter (1942) afirma que en la realidad lo que cuenta del sistema capitalista no es la competencia en precios, sino la competencia por parte de nuevos bienes, nuevas tecnologías, nuevas fuentes de oferta y nuevos tipos de organización. Se trata de una competencia que supone ventajas decisivas en costes y calidades, que no afecta simplemente a la producción y a los beneficios marginales de las empresas presentes en el mercado, sino a los mismos supuestos de su supervivencia.

El capitalismo no se mueve gracias a consumidores que eligen marginalmente entre productos preexistentes para igualar la relación entre utilidades marginales a la relación entre precios paramétricos, o gracias a empresarios que coordinan factores de producción homogéneos, sino por las presiones de la «destrucción creativa». Se trata de un proceso de cambio industrial que revoluciona incansablemente la estructura económica desde su interior, destruyendo la antigua y creando una nueva. El proceso de destrucción creativa es un hecho incontestable del capitalismo.

Según Schumpeter (1942), la sociedad estacionaria, en la que no hay más que trabajo rutinario tanto por parte de los trabajadores como de los empresarios, no puede describir de forma adecuada todo el proceso económico, porque la historia del capitalismo está marcada por «explosiones y catástrofes violentas», que nos debería impulsar a descartar la idea de que éste sea un proceso harmónico y estable. Estos desequilibrios - los ciclos económicos- se generan como consecuencia de la acción dinámica de los empresarios innovadores que implementan nuevas combinaciones económicas - nuevos productos, nuevos procesos de producción, nuevas formas de organización, nuevas fuentes de materias primas e incluso nuevos mercados.

Estos nuevos empresarios - los emprendedores schumpeterianos- transforman las invenciones en innovaciones, haciendo que las invenciones sean explotables desde un punto de vista económico, es decir actúan como nexo entre el avance de la ciencia y la tecnología y el progreso económico. El riesgo al que se enfrentan estos homines novi se ve compensado por los beneficios que les proporciona, temporalmente, la renta monopolística generada por la innovación. 
Esta temporalidad está determinada por el hecho de que rápidamente la innovación será imitada y perfeccionada por la competencia. Este hecho favorecerá el desarrollo de nuevos avances técnicos que a su vez volverán a desequilibrar el sistema. A lo largo de este proceso, el dinamismo de las inversiones empezará a mostrar signos de agotamiento, a la vez que la demanda de los productos se debilitará, reforzando el agotamiento de la innovación. Al final del proceso de imitación-difusión, la innovación ya no será tal y el sistema volverá a seguir las pautas del camino marcado por el equilibrio estacionario, hasta que una nueva innovación vuelva a abrir el ciclo que marcará el proceso de crecimiento del capitalismo.

En Schumpeter (1939), para comprender cómo el capitalismo crea nuevas estructuras industriales, hay que ver a la empresa como un agente del cambio y al emprendedor como fuente de ideas nuevas. En la perspectiva schumpeteriana, el papel que juega el emprendedor es crucial, ya que es el origen de las nuevas ideas y el que va a llevarlas a cabo. Su función no es maximizar los beneficios sustituyendo marginalmente entre factores de producción homogéneos en el ámbito de la producción de productos dados y conocidos. Su función es la de revolucionar los métodos productivos y las formas organizativas existentes y la de introducir nuevos productos por medio de la creación de nuevas empresas. Asimismo, la introducción de productos verdaderamente nuevos supone el nacimiento de nuevos mercados.

El nivel de eficiencia económica del sistema mejora gracias a cambios discontinuos que maduran dentro de él. La ruptura del equilibrio adquiere unas connotaciones positivas en cuanto nos encontramos en una óptica dinámica que supone la posibilidad de mejorar las condiciones de eficiencia del sistema a través de la innovación y del progreso generados por las empresas, que ya no son externos a su función de producción: pensemos en la introducción de nuevos productos, al uso de tecnologías que ahorren recursos, a la mejora de la calidad de los productos mismos. Por extensión, también el concepto estático de óptimo paretiano ya no constituye un objetivo a perseguir, en cuanto sinónimo de estancamiento.

El mercado puede definirse cómo el espacio en el que tiene lugar la confrontación entre empresas que compiten en sentido dinámico, es decir, empresas que se esfuerzan para emerger por medio 
de la oferta de productos cada vez mejores, de modelos de negocio nuevos y más eficaces, y del aumento de la productividad. En otras palabras, el mercado es el lugar en el que se materializa la distribución de "premios» - beneficios altos y mayores cuotas de mercado- a los mejores, y de «castigos» — beneficios bajos o pérdidas y reducción de la cuota de mercado- para los más ineficientes. El castigo máximo es la quiebra, que conlleva el abandono del mercado competitivo. Así pues, el sistema de mercado constituye un mecanismo de incentivos y selección, que conduce de manera inexorable al fortalecimiento de las empresas más hábiles, capaces de innovar a nivel organizativo, de procesos y de productos.

En términos de eficiencia, la competencia, actuando en un contexto dinámico e incierto, desempeña un papel constante de selección de las empresas, influyendo en su capacidad de minimizar los costes y de innovar productos y procesos productivos. Sin embargo, en términos de eficiencia, los efectos de una mayor competencia no se caracterizan por su carácter unívoco. De hecho, las empresas más grandes pueden generar una mayor innovación tanto de proceso como de producto. Además, la perspectiva de conseguir un poder de mercado y de poder aplicar un precio de monopolio supone un incentivo fundamental para que las empresas innoven, ya que confían en las rentas monopolísticas que podrán obtener una vez introducidas las innovaciones - mecanismo entre otros que se utiliza para justificar la existencia de las patentes.

Así pues, según Schumpeter (1942), la imperfección de la competencia, con la presencia de elementos de monopolio, es necesaria para permitir la apropiación de los beneficios de la innovación y, por lo tanto, para estimular el progreso tecnológico, que está al origen del extraordinario aumento del bienestar que se ha producido a partir de la primera revolución industrial. Las empresas que compiten en el marco de unos mercados no perfectamente competitivos son siempre distintas las unas de las otras. Un equilibrio en el cual todas son iguales es totalmente inconcebible, porque cada empresa incorpora conocimientos específicos idiosincrásicos, que son el resultado de una historia pasada que las hace diferentes de todas las demás. La existencia de beneficios extraordinarios - llamémosles, por razones de simplicidad, beneficios o, más correctamente, cuasi-rentas - es también normal y se deriva del hecho de 
que las empresas son «más o menos capaces», tanto en el sentido de ser más o menos capaces de desempeñar eficientemente sus actividades tradicionales, como en el sentido de ser más o menos capaces de ocupar áreas de mercado en rápido crecimiento o incluso de crear otras nuevas.

Desde esta perspectiva, la transición desde el monopolio a la libre competencia no implica necesariamente beneficios para todas las generaciones, presentes y futuras. De hecho, sólo las generaciones presentes se beneficiarán con seguridad, en cuanto que el monopolio permite un mayor crecimiento de la economía. En otras palabras, la competencia perfecta permite que las generaciones presentes mejoren con respecto a la misma situación en la que haya un monopolio, pero las generaciones futuras podrían empeorar, debido a que la ausencia de beneficios marginales impediría la financiación de las inversiones en investigación y desarrollo. Ello a su vez constreñiría todo estímulo a la innovación y a la mejora del nivel cualitativo de bienes y servicios, limitando así el crecimiento de la eficiencia en el tiempo. Por lo tanto, el crecimiento económico asociado a regímenes de oligopolio o monopolio podría ser más sostenido en comparación al crecimiento asociado a situaciones de competencia perfecta.

En la visión schumpeteriana, en los cimientos del crecimiento económico se encuentra la innovación que, por definición, requiere inversiones arriesgadas por parte de las empresas. La financiación de las inversiones en investigación es onerosa y los intermediarios financieros son bastante reticentes a la hora de financiar proyectos cuyo rendimiento esperado está sujeto a un importante nivel de riesgo. Por esta razón, la fuente principal de financiación de las inversiones en investigación es la financiación propia. Ahora bien, puesto que las empresas en monopolio obtienen unos beneficios más elevados que en competencia perfecta, es razonable creer que así podrán destinar mayores recursos para financiar la investigación, generando mayores descubrimientos y mejores innovaciones, y por ende garantizando un crecimiento más rápido.

Según Schumpeter (1942), es precisamente la expectativa de poder constituir un monopolio y de disfrutar de sus rentas lo que impulsa la inversión en investigación de las empresas. Si las empresas supieran que las rentas del monopolio son breves y de al- 
cance reducido, no emprenderían ningún tipo de esfuerzo en investigación y desarrollo, y no desencadenarían el proceso de crecimiento. Así pues, la existencia de monopolios es beneficiosa a largo plazo tanto porque impulsa las inversiones de las empresas como porque les proporciona los recursos adecuados.

III

\section{EL DEBATE POST-SCHUMPETERIANO}

A las ideas de Schumpeter se le suele contraponer la visión antagónica de Arrow quien, en un conocido escrito de 1962, contestó la idea de que los monopolios puedan ser eficientes en sentido dinámico y trató de argumentar que la competencia no sólo garantiza la eficiencia estática, sino también una tasa de crecimiento económico más elevada con respecto a una situación de monopolio. La idea básica de Arrow puede resumirse en dos simples proposiciones. Por un lado, quienes disfruten de rentas de monopolio no tienen incentivos para realizar actividades de investigación y desarrollo -y por lo tanto no generan crecimiento. Por otro, los monopolios suelen asociarse a situaciones en las que la información sobre la tecnología está protegida por patentes y por lo tanto se difunde con dificultad, ralentizando el proceso de crecimiento que por el contrario se basa en las posibilidades de utilizar, conocer y mejorar las tecnologías de las empresas presentes en el mercado.

Estas posiciones enfrentadas han dado lugar a un largo debate —conocido como «Schumpeter versus Arrow»— en el que el problema central se convirtió en establecer si se realiza un mayor esfuerzo en investigación y desarrollo —es decir, si se invierte más- en aquellos sectores donde prevalecen situaciones de monopolio o donde prevalecen condiciones de competencia. La contraposición reside en una perspectiva temporal diferente a las que los dos autores hacen referencia. En una visión ex ante, es prioritario salvaguardar los incentivos de las empresas a realizar actividades de investigación, que están mejor garantizados por una situación de monopolio o, dicho de otro modo, de poder de mercado, en la cual la empresa podrá apropiarse de los beneficios generados por sus inversiones (Gilbert y Newbery, 1982; Grossman y Helpman, 1991; Aghion y Howitt, 1998). 
Por el contrario, en una visión ex post, es deseable que, una vez introducida una innovación, todas las empresas presentes en el mercado puedan tener acceso a ella (Louly, 1979; Dasgupta y Stiglitz, 1980).

Una valoración exhaustiva de las teorías de Schumpeter y Arrow no puede, sin embargo, prescindir del hecho de que ambos autores evidentemente elaboran su pensamiento en un contexto histórico en el que la realidad económica era muy distinta de la actual. Las inversiones no exigían las aportaciones de capital que se requieren en la actualidad para poder desarrollar actividades empresariales innovadoras. Además, las que entonces eran situaciones de monopolio, en la actualidad, tras la ampliación de los mercados vinculada a la globalización, se corresponden a situaciones cuasi-competitivas en las que las rentas del monopolio se erosionan mucho más rápidamente como consecuencia de la competencia internacional.

Aunque la dinámica de la innovación y los mecanismos que la generan hayan evolucionado en sus términos de referencia, los fundamentos teóricos en los que se basan una y otra posición tienen sus propios seguidores y detractores, y por lo tanto el debate debe de considerarse todavía abierto. Los estudios que se han enfrentado al problema desde el punto de vista de la evidencia empírica, no presentan unos resultados unívocos. De hecho, hay aportaciones académicas que detectan una mayor intensidad de las inversiones en actividades de investigación en aquellos sectores en los que prevalecen las condiciones de monopolio, y otras, por el contrario, que alcanzan unas conclusiones opuestas, es decir, que las inversiones en actividades de investigación son mayores en los sectores más competitivos (Aghion y Howitt, 1998).

La literatura más reciente (Aghion et al., 2005) propone una solución intermedia, caracterizada por un nivel suficiente de competencia asociado a un poder de mercado significativo de las empresas, que parecería ser la más adecuada para asegurar la continuidad de la innovación y, en particular, de la investigación tecnológica. En otras palabras, existiría una relación en forma de «U» invertida entre competencia e innovación, tomada como proxy de la eficiencia dinámica. En una primera fase, a medida que aumenta la primera, también aumenta la segunda, pero no de manera proporcional. Superado el punto de máximo, la competencia se vuelve demasiado intensa, reduciendo los incentivos a invertir y a innovar. 
El debate abierto por Schumpeter sobre los beneficios de la concentración del mercado de cara a las actividades de innovación, también puede analizarse de desde el enfoque de la teoría de la elección pública. La existencia de estructuras de mercado monopolísticas generadas en base a un proceso innovador ha sido vista siempre con un cierto recelo por parte de políticos y burócratas que han encontrado en ello la justificación para desarrollar marcos normativos y reguladores dirigidos a intervenir el libre funcionamiento del mercado. Desde un punto de vista normativo, este esquema de funcionamiento ha sido rebatido de forma contundente por la escuela de la Public Choice, que ha argumentado persuasivamente cómo los fallos de la intervención pública pueden ser más perjudiciales que los fallos del mercado de la economía ortodoxa. Buchanan y Tullock (1962) demostraron claramente que la intervención pública no persigue necesariamente el bienestar colectivo. Según la visión del public choice, precisamente porque actúan según modalidades neoclásicas, el político y el burócrata maximizarán su propio interés personal. Aunque en ocasiones dicho interés personal pueda coincidir con el de la colectividad, en la mayoría de los casos esto no suele suceder. Por lo tanto, existen razones más que suficientes para considerar cuidadosamente toda regulación cuya finalidad sea modificar artificialmente las acciones de las empresas privadas, o incluso la creación de nuevas empresas, como puede ser el caso del empresario público o del político con aspiraciones redistributivas. Mientras que los errores del empresario privado tienen su frontera en el beneficio negativo, dicha frontera es mucho más difusa en el caso del agente público, que puede contar con la tolerancia de los contribuyentes y con el hecho de que raramente las elecciones de voto del electorado están determinadas por unas pocas decisiones específicas del político de turno.

IV

LA RESPUESTA DE LA ESCUELA AUSTRIACA DE ECONOMÍA. EL EMPRESARIO COMO CLAVE DEL PROCESO INNOVADOR

La respuesta a este debate la proporciona la teoría de la Escuela Austriaca, con su visión dinámica de la competencia. Como apun- 
taba Hayek (1948) cuando distinguía entre competencia perfecta y competencia dinámica, en una perspectiva dinámica, el equilibrio estable del mercado es inalcanzable, por lo que hay que fijarse en la interacción continua entre empresario y entorno económico. Es el empresario quien, gracias a su poder de mercado, coordina y modifica dicho entorno. Además, los costes, los precios y el comportamiento del mercado se manifiestan a través del proceso competitivo, agrupando toda una serie de informaciones dispersas e imperfectas. Por ejemplo, las empresas no conocen el valor del coste mínimo de producción - cada empresa tiene un coste diferente- pero lo pueden inferir gracias a la evidencia del mercado, en el cual podrán constatar la mejor oferta de sus competidores.

Por lo tanto, la contribución a la eficiencia de la competencia está vinculada a la trasmisión de la información, que de lo contrario estaría dispersa entre los distintos agentes, y permite así su mejor aprovechamiento. Por otra parte, precisamente porque los empresarios mantienen la información relevante de forma extremadamente desagregada y descentralizada, ésta tampoco puede ser determinada de forma alternativa por medio de simulaciones de mercado llevadas a cabo por jueces o administradores centralizados.

Por estas razones, Hayek (1948) y Mises (1949) rechazaron tanto la prohibición de trust y cárteles, así como el control de las políticas empresariales perseguidas por agentes económicos con poder de mercado, a causa de sus efectos negativos sobre el sistema de incentivos que genera el proceso de competencia dinámica. Las restricciones y las limitaciones de la competencia llevadas a cabo por las empresas se ven considerablemente reducidas gracias al papel que juega la competencia potencial. El análisis de la competencia tradicional se basa en la competencia efectiva en el mercado, aunque la competencia puede existir también a nivel latente o potencial, lo cual impide el aprovechamiento de posiciones de monopolio, por lo menos a largo plazo. La idea de la competencia potencial encontró una posterior sanción por parte de la Escuela de Chicago y con la formalización de la teoría de los mercados contestables o mercados disputados. Ambos planteamientos destacan la importancia de la competencia potencial como freno a la conducta abusiva de los monopolios. 
La teoría del empresario innovador de la Escuela Austriaca se diferencia de la visión schumpeteriana en que el empresario es innovador en cuanto consigue aprovechar oportunidades ya presentes en el mercado y explotarlas en su propio beneficio, mientras que para Schumpeter el emprendedor es aquél que crea nuevas formas de innovación. Además, el empresario austriaco actúa en un mercado imperfecto $\mathrm{y}$, aprovechando las oportunidades generadas por esta imperfección, guía el mercado hacia una nueva situación de equilibrio. El emprendedor schumpeteriano, por el contrario, hace exactamente el camino opuesto, activando un proceso de destrucción creativa.

En conclusión, el concepto de eficiencia definido por la Escuela Austriaca encuentra su explicación en la evolución de los mercados en el tiempo, en el contexto de una visión cíclica del desarrollo económico. La singularidad de esta idea reside en considerar las variables del cambio tecnológico y de la innovación como endógenas, y que están vinculadas directamente con la actividad del empresario, cuyo papel es por lo tanto decisivo a la hora de estimular el sistema económico. Por lo tanto, si Schumpeter pone de actualidad la figura del empresario, es la Escuela Austriaca la que le coloca en el mismo centro de la economía de mercado, en la que no se limita a ser un simple ejecutor de un esquema predeterminado, sino que se transforma en el descubridor por excelencia: aquél que aprovecha las oportunidades que otros habían descuidado y que hace posible la definición de nuevas producciones, nuevas tanto en términos de productos como de características funcionales.

\section{REFERENCIAS BIBLIOGRÁFICAS}

Aghion, P. et alia (2005): «Competition and Innovation: an Inverted-U Relationship», The Quarterly Journal of Economics, 120 (2), pp. 701-728.

— ; P. HowitT (1998): Endogenous Growth Theory, MIT Press, Cambridge, Mass.

ArRow, K. (1962): «Economic Welfare and the Allocation of Resources for Invention», en Groves H.M (ed.), The Rate and Direction of 
Inventive Activity: Economic and Social Factors, Princeton University Press, Princeton, pp. 609-626

Baumol, W.J., J.C. Panzar y E.D. Willig (1982): Contestable Markets and Theory of Industrial Structure, Harcourt Brace Jovanovich, New York.

Buchanan, J.M. y G. Tullock (1962): The Calculus of Consent. Logical Foundations of Constitutional Democracy, University of Michigan Press, Ann Arbour.

Dasgupta, P. y J. Stiglitz (1980): «Industrial Structure and the Nature of Innovative Activity», The Economic Journal, Vol. 90.

Gilbert, R. y D. Newbery (1982): «Preemptive Patenting and the Persistence of Monopoly», American Economic Review, Vol. 73 (3).

Grossman, G. y E. HelpMAn (1991): Innovation and Growth in the Global Economy, MIT Press, Cambridge Mass.

HAYEK, F. (1960): The Constitution of Liberty, University of Chicago Press, Chicago.

- (1948): «The meaning of competition», en F. Hayek, Individualism and Economic Order, Routledge, London.

- (1945): «The Use of Knowledge in Society», American Economic Review, n. 35, pp. 519-530

Huerta De Soto, J. (2004): «La teoría de la eficiencia dinámica», Procesos de Mercado, vol. 1 (1), pp. 11-71.

LouLY, G. (1979): «Market Structure and Innovation», Quarterly Journal of Economics, Vol. 93(3).

MisEs, L. (1949): Human Action: A Treatise of Economics, Yale University Press, Yale.

SCHERER, F.M. (1970): Industrial Market Structure and Economic Performance, Rand McNally, Chicago.

SCHUMPETER, J.A. (1942): Capitalism, socialism and democracy, Harper \& Row, New York.

— (1939): Business Cycles, McGraw-Hill, New York. 